PALEO

Revue d'archéologie préhistorique

$14 \mid 2002$

Varia

\title{
Révision du sexe du squelette magdalénien de Saint-Germain-la-Riviere (Gironde, France)
}

Sex diagnosis of the magdalenian skeleton from Saint-Germain-la-Rivière

(Gironde, France)

D. Henry-Gambier, J. Bruzek, P. Murail et F. Houët

\section{OpenEdition}

Journals

Édition électronique

URL : http://journals.openedition.org/paleo/1434

DOI : $10.4000 /$ paleo.1434

ISSN : 2101-0420

Éditeur

SAMRA

Édition imprimée

Date de publication : 1 décembre 2002

Pagination : 205-212

ISSN : 1145-3370

Référence électronique

D. Henry-Gambier, J. Bruzek, P. Murail et F. Houët, « Révision du sexe du squelette magdalénien de Saint-Germain-la-Riviere (Gironde, France) », PALEO [En ligne], 14 | 2002, mis en ligne le 16 août 2010, consulté le 26 juillet 2020. URL : http://journals.openedition.org/paleo/1434 ; DOI : https://doi.org/ 10.4000/paleo.1434

Ce document a été généré automatiquement le 26 juillet 2020.

\section{(c) (1) $(9 \Theta$}

PALEO est mis à disposition selon les termes de la licence Creative Commons Attribution - Pas d'Utilisation Commerciale - Pas de Modification 4.0 International. 


\title{
Révision du sexe du squelette magdalénien de Saint-Germain-la- Riviere (Gironde, France)
}

\author{
Sex diagnosis of the magdalenian skeleton from Saint-Germain-la-Rivière \\ (Gironde, France)
}

D. Henry-Gambier, J. Bruzek, P. Murail et F. Houët

Nous remercions J.-J. Cleyet-Merle, conservateur du Musée National de Préhistoire (Les-Eyziesde-Tayac) qui nous a autorisé à examiner le squelette de Saint-Germain-la-Rivière.

\section{Introduction}

1 La détermination du sexe constitue un aspect capital de l'étude des populations anciennes. Elle représente un paramètre majeur des interprétations paléobiologiques, paléodémographiques et culturelles, comme les pratiques funéraires par exemple.

2 Durant la dernière décennie, le paradigme de la diagnose du sexe en paléoanthropologie a changé. Les méthodes de détermination sur le crâne ont été abandonnées pour des méthodes fondées sur l'os coxal, considéré, en raison de son implication dans la reproduction, comme le plus pertinent (Bruzek 1991; Bone 1993). Dans le cas des fossiles du Paléolithique supérieur, l'état de conservation de l'os coxal a cependant constitué un frein à cette évolution méthodologique. La quasi-totalité des attributions sexuelles des adultes du Paléolithique supérieur demeure donc établie sur le crâne (Ferembach et al. 1979) et/ou sur le degré de robustesse des os du squelette post-crânien. Lorsque l'os coxal est considéré, les méthodes les plus employées sont celles de Sauter et Privat (1954 - 1955), Phenice (1969) dont la fiabilité a été remise en cause (Bruzek 1996 ; Bruzek 2002).

3 Ainsi, la morphologie de l'os coxal du squelette gravettien/épigravettien (Henry Gambier et al. 2001) de "l'homme de Menton" découvert par E. Rivière à la fin du XIXème siècle, a été révisée selon la méthode mise au point par J. Bruzek (Bruzek 1991). 
Cette analyse a conduit à attribuer un sexe féminin à ce squelette (de Lumley et al. 1992). Cela démontre bien le piège que constitue l'utilisation de critères de robustesse du crâne ou des os longs pour déterminer le sexe de spécimens issus de populations anciennes. En ce qui concerne le squelette de Saint-Germain-la-Rivière (Gironde, France), l'attribution de celui-ci à un jeune adulte de sexe féminin par H. V. Vallois, repose sur la morphologie du crâne et sur la gracilité des os longs (Blanchard et al. 1972). Cette interprétation a été soutenue par P. Guth (1973) sur la valeur $(32,5 \mathrm{~mm})$ de la largeur acétabulo-ischiatique (Sauter et Privat 1954-1955) de l'os coxal gauche ainsi que sur celle de l'angle sous-pubien $\left(100-105^{\circ}\right)$ et sur celle de l'indice ischio-pubien ( $\mathrm{I}=113$ ) de Schultz (1930).

4 En raison du peu de fiabilité de ces méthodes, il est apparu nécessaire de reprendre l'analyse du bassin de ce sujet afin de confirmer ou d'infirmer cette attribution sexuelle en appliquant les nouvelles méthodologies mises au point à partir d'un échantillon important de squelettes de populations actuelles d'âge et de sexe connus.

5 Cette première note s'inscrit dans le cadre d'un projet plus vaste qui vise à reprendre l'estimation du sexe de l'ensemble des fossiles du Paléolithique supérieur d'Europe. Elle répond aussi au souci de fournir une information fiable sur le squelette de SaintGermain-la-Rivière, l'une des pièces majeures des collections du Musée National de Préhistoire, alors qu'une nouvelle présentation muséographique de ses collections est en cours de réalisation.

\section{Méthodes}

Deux approches ont été mises en œuvre, la première morphologique et la seconde morphométrique. Toutes deux reposent sur une élaboration méthodologique mettant en jeu un échantillon de référence important quantitativement et représentatif de la variabilité mondiale actuelle. Ces méthodes ont été testées sur d'autres échantillons de sexe connu et leur fiabilité est attestée. Enfin, elles s'appuient sur la notion de compensation du dimorphisme sexuel entre les différents segments morpho fonctionnels de l'os coxal, impliquant ainsi plusieurs variables qualitatives ou quantitatives permettant de mieux circonscrire la sexualisation de l'os coxal dans sa globalité.

\section{Approche morphologique}

7 La méthode définie par J. Bruzek $(1991 ; 2002)$ s'appuie sur une évaluation morphoscopique de la forme sexuelle de cinq critères morpho-fonctionnels. Sa fiabilité est de $95 \%$.

\section{Approche morphométrique}

8 Il a été récemment démontré qu'il était possible de définir, à partir des dimensions de l'os coxal, un dimorphisme sexuel commun chez Homo sapiens sapiens, quelle que soit la population considérée (Bruzek et al. 1999 ; Houët et al. 1999). Nous avons donc comparé la combinaison des dimensions de l'os coxal gauche, mieux conservé, à un large échantillon de référence de sexe connu incluant des populations d'Europe, d'Amérique du Nord, d'Afrique et d'Asie (2). L'estimation du sexe est obtenue à partir des 
probabilités a posteriori (associées aux distances de Mahalanobis du spécimen par rapport aux groupes masculins et féminins de référence). Cette démarche statistique, qui met en jeu l'analyse discriminante, a déjà été démontrée comme très performante dans le cadre de la diagnose sexuelle (Murail et al. 1999).

\section{Matériel}

Le squelette de Saint-Germain-la-Rivière découvert le 15 décembre 1934 par R. Blanchard (Blanchard 1935) dans un abri sous roche situé en rive droite de la Dordogne constitue pour le Paléolithique supérieur un document exceptionnel. Associé à des artéfacts de facture magdalénienne (Blanchard et al. 1972), le corps ocré et accompagné d'une riche parure de coquillages et de canines de Cervidé percées et gravées, reposait sur le côté gauche, membres supérieurs et inférieurs fléchis, vraisemblablement dans une fosse creusée jusqu'au substrat calcaire. Les parois de cette fosse colmatée après le dépôt du cadavre, étaient étayées par des dalles. Deux autres dalles posées sur ces blocs complétaient la structure (Blanchard et al. 1972; Gambier 1990). Une côte de ce squelette a été récemment datée par la méthode du C14 en SMA. Le résultat - GifA 95456, $15780 \pm 200$ BP (Gambier et al. 2000) - est cohérent avec une attribution au Magdalénien moyen.

10 Le squelette est presque complet. Le bassin est représenté par les deux os coxaux et une partie du sacrum (fig. 1). La surface des deux os est ocrée et encore encroûtée de sédiment par endroit. L'os très friable a été verni et des traces de restaurations anciennes réalisées avec du plâtre ou des résines sont visibles en plusieurs points.

\section{Estimation de l'âge au décès}

11 Toutes les épiphyses des os longs sont fusionnées aux diaphyses. On note aussi la fusion complète de la crête iliaque, de la tubérosité ischiatique pour l'os coxal ainsi que celle de l'épiphyse médiale de la clavicule. Ces caractéristiques indiquent un sujet adulte.

12 Sur l'os coxal, les crêtes et les sillons de la surface de la symphyse pubienne sont bien définis et orientés perpendiculairement à l'axe longitudinal. Le rempart n'est pas formé et la lèvre postérieure ne "déborde" pas. Ces traits sont conformes au stade I défini par A. Schmitt (2001) et plaident en faveur d'un jeune adulte.

13 Ces données sont compatibles avec l'âge dentaire. Sur l'os maxillaire comme sur la mandibule, la troisième molaire n'a pas achevé son éruption et sa surface occlusale n'atteint pas le niveau de celle de la seconde molaire (observation D. Henry - Gambier).

\section{État de conservation des deux os coxaux}

\section{Os coxal droit (fig. 1, 2 et 3 )}

Il comprend l'ilium pratiquement complet. Une cassure ancienne allant d'un point situé entre le bord acétabulaire et l'épine iliaque antéro-inférieure à un point de la crête iliaque situé à environ $10 \mathrm{~cm}$ de l'épine iliaque postéro-supérieure est observable. Cette cassure se traduit sur la face antéro-médiale par une fissure de 2 à $3 \mathrm{~mm}$. Sur la face postéro-latérale, la trace de cette cassure est masquée par le vernis. 
15 L'état de conservation du pubis est moins bon (fig. 5). Le tuberculum pubicum et la partie inférieure de la branche descendante manquent. La partie inférieure de la symphyse pubienne est érodée. La partie du pubis qui participe à l'acetabulum est détruite et comblée par du plâtre.

16 L'os ischium est limité à la partie acétabulaire. Séparée à l'origine du reste de l'os, elle a été recollée de telle sorte que la surface dorsale située au-dessus du tuber ischiadicum offre une morphologie anormale. De ce fait, la grande incisure ischiatique et le foramen obturatum sont incomplets.

Fig. 1 - Squelette de Saint-Germain-la-Rivière : Vue supérieure du bassin Fig. 1 - Saint-Germain-la-Rivière skeleton: Superior view of the hip bone

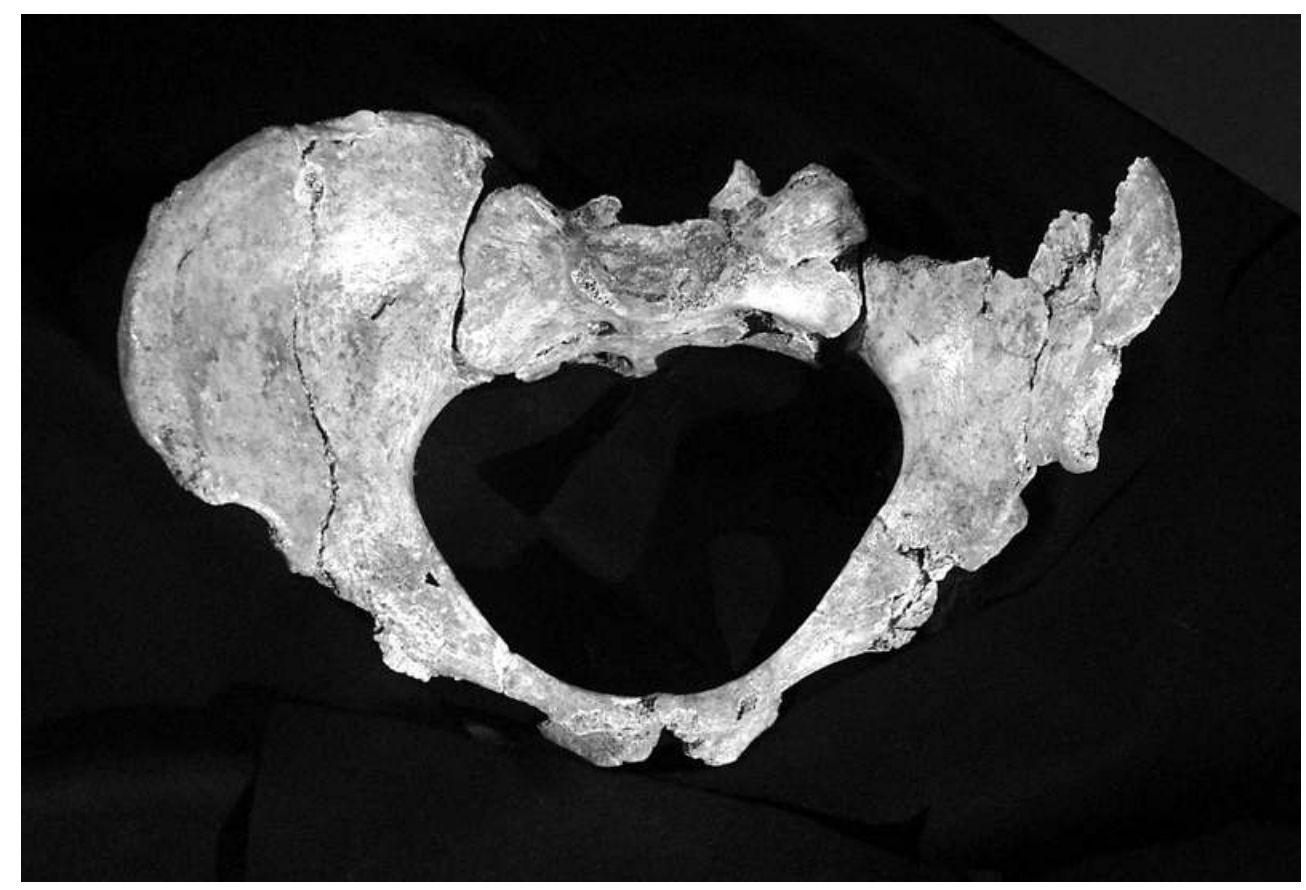


Fig. 2 - Squelette de Saint-Germain-la-Rivière : Vue médiale de l'os coxal droit Fig. 2 - Saint-Germain-la-Rivière skeleton : Medial view of the right coxal bone

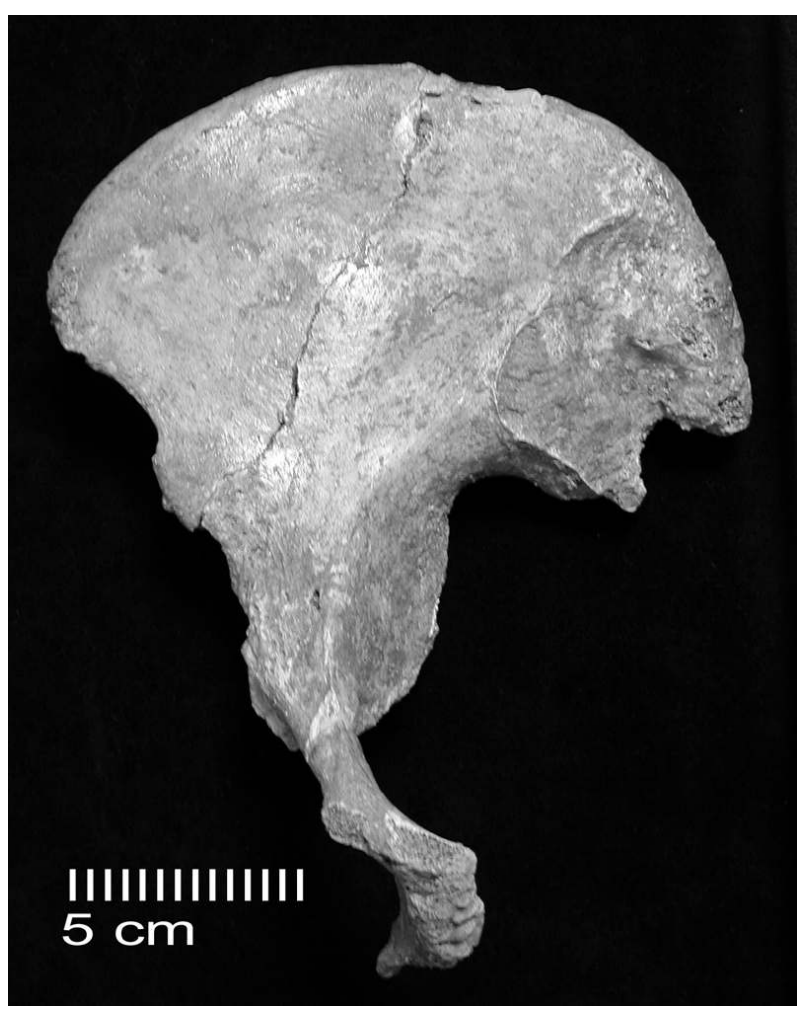

Fig. 3 - Squelette de Saint-Germain-la-Rivière : Vue latérale de l'os coxal droit Fig. 3 - Saint-Germain-la-Rivière skeleton : Lateral view of the right coxal bone

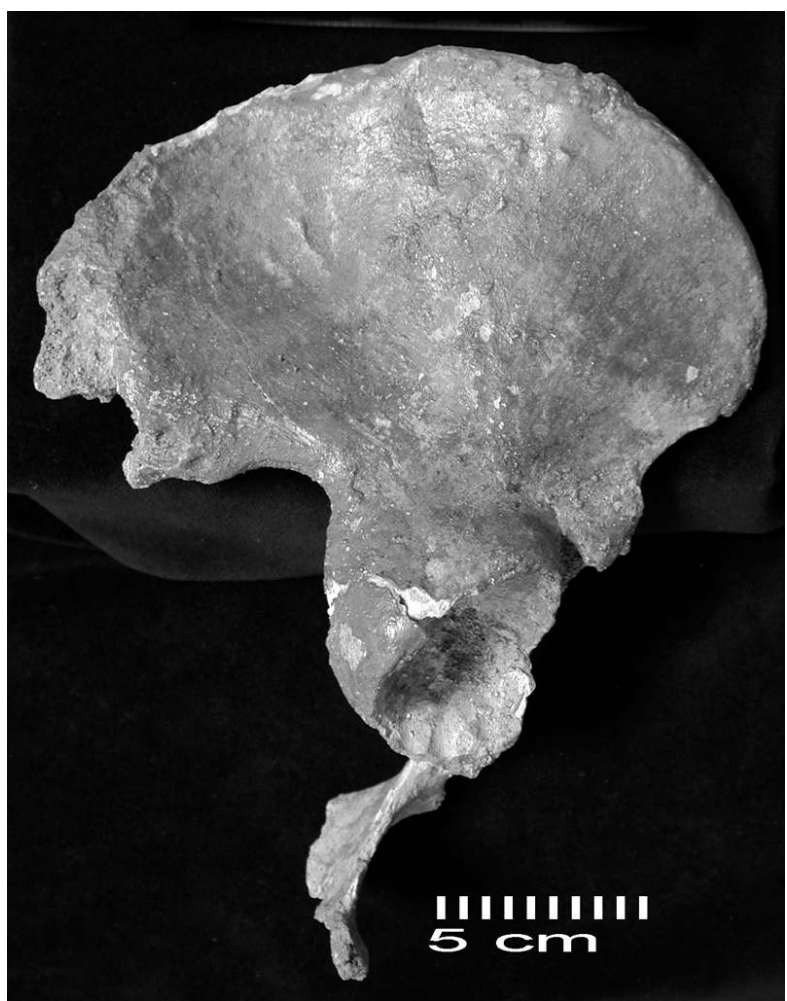




\section{Os coxal gauche (fig. 4)}

17 L'ilium droit est plus endommagé que le gauche; en revanche la région ischio-pubienne est mieux représentée. Le tiers antérieur de l'aile iliaque et le segment antérieur du facies auricularis sont présents. Les deux tiers postérieurs de l'aile iliaque et la moitié postérieure de la surface sacro-pelvienne manquent.

18 Le secteur conservé de l'aile iliaque est constitué de quatre fragments associés par collage. Sur la face antéro-médiale, ils sont séparés par des lacunes osseuses (1 à $2 \mathrm{~mm}$ ). Sur la face postéro-latérale, le contact entre les fragments est bon.

19 Le pubis est pratiquement complet, tuberculum pubicum inclus. La reconstitution du bord antérieur de l'acetabulum à partir de plusieurs esquilles d'os plus ou moins jointives n'est cependant pas totalement satisfaisante.

20 L'ischium est très bien préservé excepté au niveau de l'épine ischiatique et du bord médial de la tubérosité ischiatique qui ne sont pas conservés.

21 Le fond de la fossa acetabuli est lacunaire se révélant ainsi comme la région la plus endommagée du segment ischio-pubien.

Fig. 4 - Squelette de Saint-Germain-la-Rivière : Vue médiale de l'os coxal gauche Fig. 4 - Saint-Germain-la-Rivière skeleton : Medial view of the left coxal bone

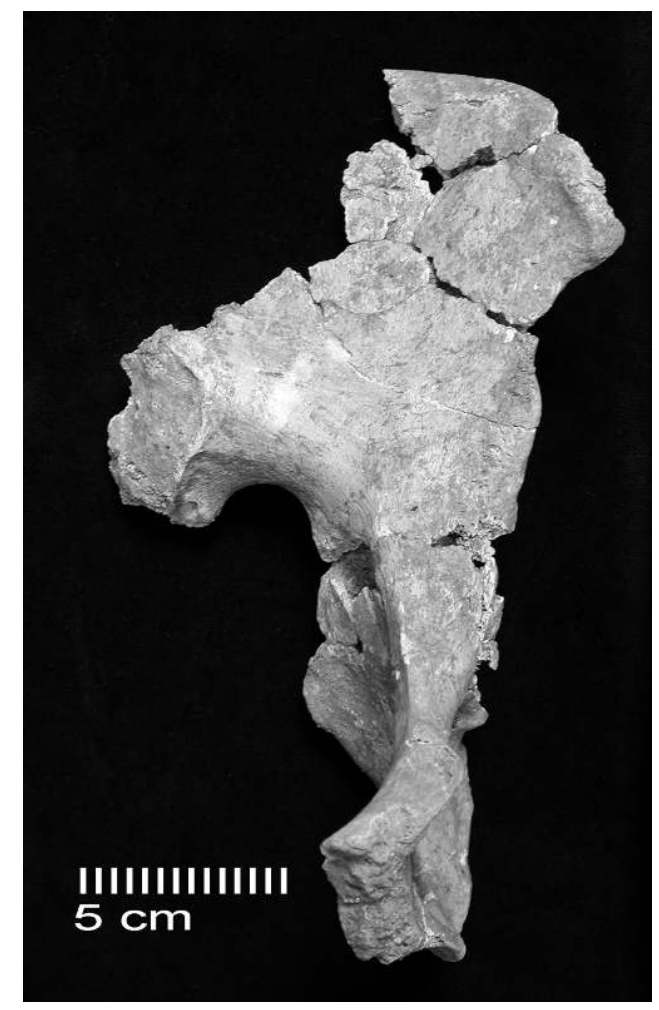

\section{Approche morphologique}

\section{Os coxal droit}

Dans la région préauriculaire, on observe une dépression profonde au contour bien délimité, associée à un tubercule (fig. 6). Il s'agit d'un sillon préauriculaire atypique 
plaidant toutefois en faveur d'une morphologie féminine. Les autres caractères de la méthode Bruzek $(1991$; 2002) ne sont pas observables en raison de l'état de conservation de l'os.

Le seul caractère de l'os coxal droit qui a pu être évalué plaide en faveur du sexe féminin.

Fig. 5 - Squelette de Saint-Germain-la-Rivière : Vue antérieure de la région pubienne de l'os coxal droit

Fig. 5 - Saint-Germain-la-Rivière skeleton : Right coxal bone frontal view of the pubis part

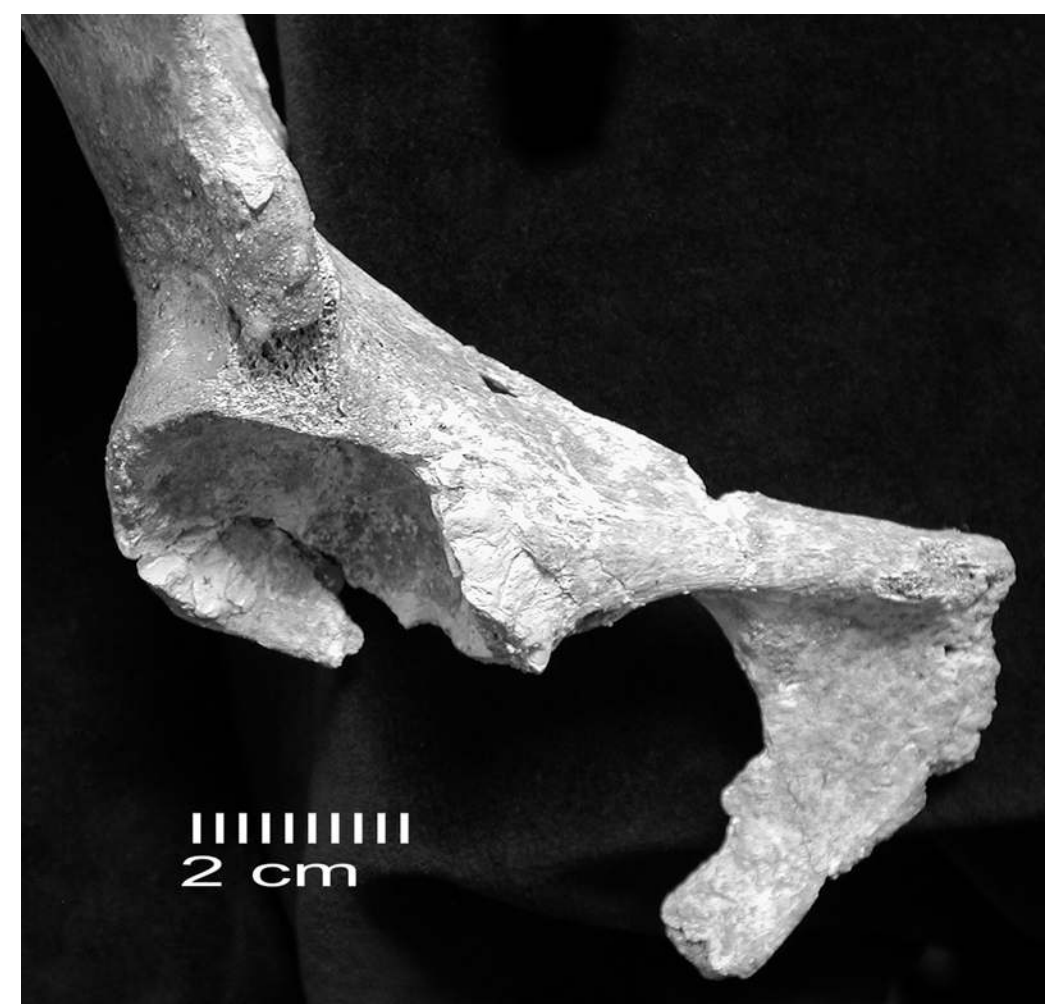


Fig. 6 - Squelette de Saint-Germain-la-Rivière : Région préauriculaire de l'os coxal droit. 1 : sillon , 2 : tubercule

Fig. 6 - Saint-Germain-la-Rivière skeleton : Preauricular surface of the right coxal bone. (1) depression ; (2) tubercle

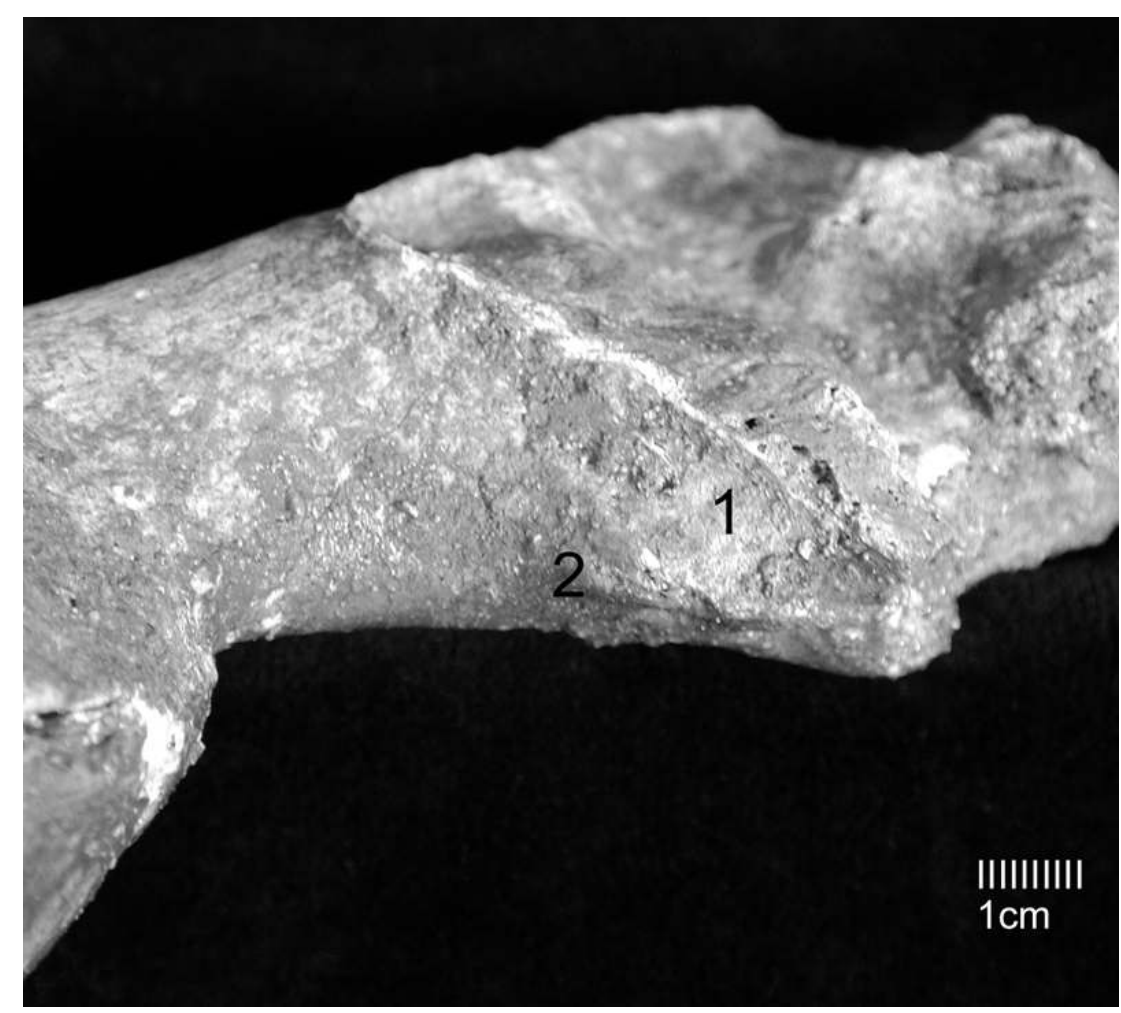

\section{Os coxal gauche}

La région préauriculaire montre une disposition particulière : un tubercule fort précède une fossette (4,5mm par $8,9 \mathrm{~mm}$ ) assez profonde (fig. 7). Il ne s'agit pas d'un foramen nourricier mais plus vraisemblablement d'un trait pathologique. Un sillon au contour délimité est visible. Un faible doute subsiste cependant car la surface de l'os est à ce niveau en partie masquée par du sédiment ocré. La forme de la grande incisure ischiatique dont l'un des segments est endommagé n'a pu être évaluée. 
Fig. 7 - Squelette de Saint-Germain-la-Rivière : Région préauriculaire de l'os coxal gauche. 1 : sillon , 2 : tubercule. Le tubercule précède une fossette

Fig. 7 - Saint-Germain-la-Rivière skeleton : Preauricular surface of the left coxal bone with pit. (1) depression ; (2) tubercle and pit

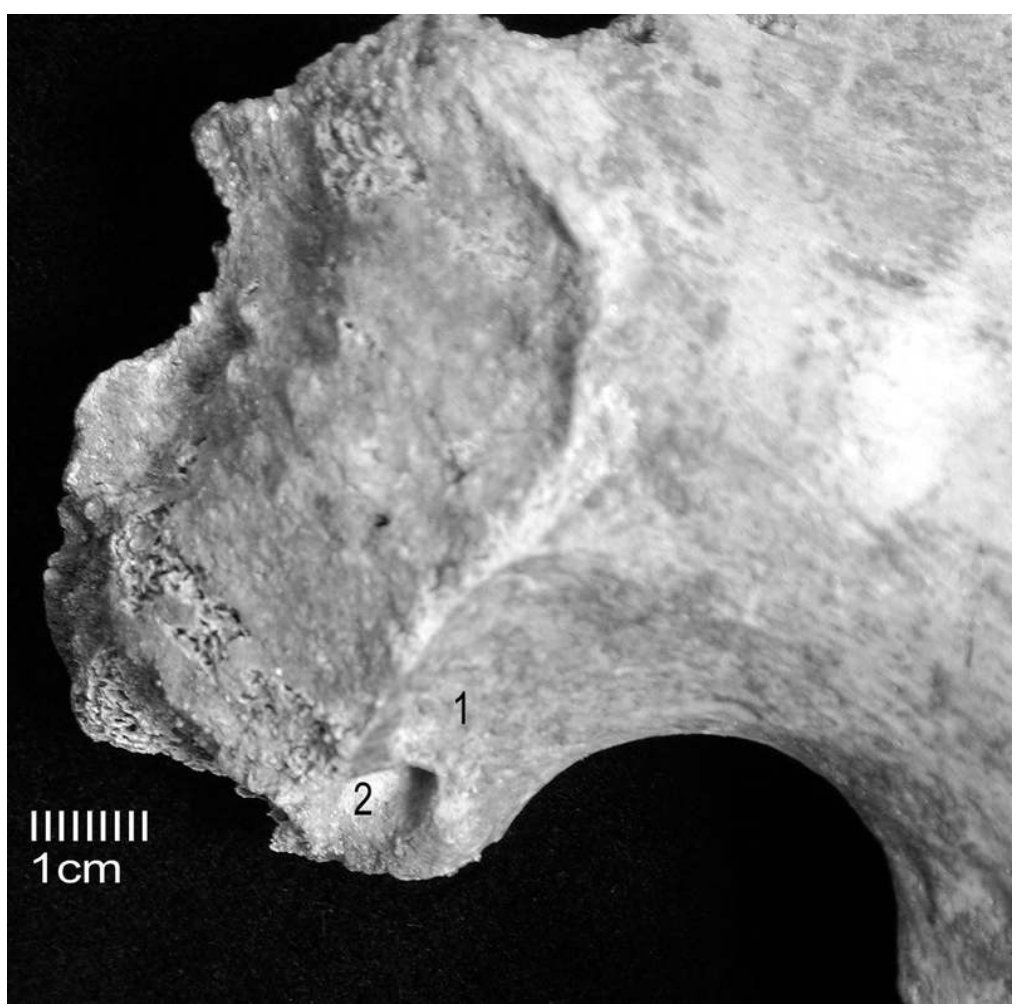

Le double arc qui forme l'arc composé est un caractère féminin. Le bord inférieur de l'os coxal est aussi nettement de morphologie féminine car sa partie centrale présente une éversion latérale. Elle est dépourvue de crista phallica et apparait dans son ensemble gracile. Les proportions ischio-pubiennes sont de type féminin avec un pubis relativement plus long que l'ischium. Pour cet os, quatre des cinq caractères pris en compte dans la méthode sont donc nettement féminins, le cinquième n'ayant pu être observé.

La morphologie des deux os coxaux du sujet de Saint-Germain-la-Rivière est ainsi sans ambiguïté féminine. En particulier, la présence d'un sillon préauriculaire même atypique pourrait témoigner de son passé obstétrical.

Tabl. 1 - Squelette de Saint-Germain-la-Rivière : os coxal gauche, dimensions en mm Table 1 - The Saint-Germain-la-Rivière skeleton: left coxal bone, measurements in $\mathrm{mm}$

\begin{tabular}{|l|l|l|l|}
\hline (Bruzek, 1991) & \multicolumn{1}{|c|}{ Os coxal gauche } & Réf. & Os coxal gauche \\
\hline PUM & Longueur du pubis préacétabulaire & M14 (Braüer, 1988) & 77,0 \\
\hline ISM & $\begin{array}{l}\text { Longueur de l'ischium } \\
\text { post - acétabulaire }\end{array}$ & (Thieme, Schull, 1957) & 88,0 \\
\hline ISMM & $\begin{array}{l}\text { Longueur de l'ischium } \\
\text { post - acétabulaire maximale }\end{array}$ & (Schulter - Ellis et al., 1985) & 104,4 \\
\hline
\end{tabular}




\begin{tabular}{|l|l|l|l|}
\hline SIS & Largeur acétabulo - ischiatique & M14 - 1(Braüer, 1988) & 32,8 \\
\hline SA & Distance spino - auriculaire & Gaillard (1960) & 76,8 \\
\hline SS & Distance spino - ischiatique & Gaillard (1960) & 66,5 \\
\hline
\end{tabular}

\section{Approche morphométrique}

Pour l'os coxal gauche, les six dimensions (tab. 1) considérées sont: la longueur préacétabulaire du pubis (Bräuer 1988), la longueur de l'ischium post-acétabulaire (Thieme et Schull 1957), la longueur de l'ischium post-acétabulaire maximale (Schulter Ellis et al. 1985), la largeur acétabulo-ischiatique, la distance spino - auriculaire et la distance spino-ischiatique (Gaillard 1960). La combinaison de ces variables dans l'échantillon de référence permet une discrimination forte (lambda de Wilks $=0,26$ ) et une fiabilité de la diagnose sexuelle très importante au seuil probabiliste de 0,95 (fiabilité $=99,4 \%, 1765$ os coxaux). Le spécimen de Saint Germain-La-Rivière est classé féminin avec une probabilité égale à 0,999 (pour le seuil probabiliste de 0,999 , le taux d'erreur est de $0 \%$ sur l'échantillon de référence).

Cela signifie que, si le dimorphisme sexuel des populations du Paléolithique supérieur ne diffère pas de celui de l'ensemble des populations actuelles, ce qui est vraisemblable en raison de leur identité taxonomique, le spécimen de Saint-Germain-la-Rivière est statistiquement féminin avec un risque d'erreur négligeable.

\section{Conclusion}

Ce réexamen des os coxaux du squelette de Saint-Germain-la-Rivière confirme de manière certaine l'attribution de ce spécimen à une jeune femme adulte.

Cette première révision illustre tout l'intérêt que présenterait l'application de ces nouvelles méthodologies à tous les spécimens des populations du Paléolithique supérieur dont l'os coxal est préservé.

\section{BIBLIOGRAPHIE}

BLANCHARD R. 1935 - Découverte d'un squelette humain à Saint - Germain - la - Rivière. Revue Historique et Archéologique du Libournais, 9, p. 11 - 18.

BLANCHARD R. , PEYRONY D.,VALLOIS H.V. 1972 - Le gisement et le squelette de Saint-Germain-laRivière. Archives de l'Institut de Paléontologie humaine, Paris, Masson.

BONES K. E. 1993 - Bias in skeleton sexing. American Journal of Physical Anthropology, supp. 16, p. 59. 
BRAUER G. 1988 - Osteometrie. In: Anthropologir, handbuch der vergleichenden Biologie des Menschen, Band I. R. Knussmann (ed.), Stuttgart: Gustav Fisher Verlag, p. 160 - 232.

BRUZEK J. 1991 - Fiabilité des procédés de détermination du sexe à partir de l'os coxal. Implications à l'étude du dimorphisme sexuel de l'homme fossile, Thèse de doctorat, Museum d'Histoire Naturelle, Paris.

BRUZEK J. 1996 - Interprétation biologique de séries archéologiques. Impact d'une diagnose sexuelle erronées à partir de simulation dans un échantillon de sexe connu. In : L. Buchet (ed.), L'identité des populations archéologiques, XVème Rencontres Internationales d'Archéologie et d'Histoire d'Antibes, Editions APDCA, Sophia Antipolis, p. 415 - 425.

BRUZEK J. 2002 - A Method for Visual Determination of Sex, Using the Human Hip Bone. American Journal of Physical Anthropology, 117, p. 157 - 168.

BRUZEK J., MURAIL P. et HOUËT F. 1999 - Diagnose sexuelle probabiliste (DSP) à partir de données métriques de l'os coxal. Bulletins et Mémoires de la Société d'Anthropologie de Paris, résumés des communications de la 1825e réunion de la Société d'Anthropologie de Paris, 11 (3 - 4), p. 484.

FEREMBACH D., SCHWIDETZKY I., STLOUKAL M. 1979 - Recommandations pour déterminer l'âge et le sexe sur le squelette. Bulletins et Mémoires de la Société d'Anthropologie de Paris, 6, 13, p. 7 - 45.

GAILLARD J. 1960 - Détermination sexuelle d'un os coxal fragmentaire. Bulletins et Mémoires de la Société d'Anthropologie de Paris, 1, 11, p. 225 - 267.

GAMBIER D. 1990 - Pratiques funéraires au Paléolithique supérieur en France : Les sépultures primaires. Bulletins et Mémoires de la Société d'Anthropologie de Paris, 2, 3 - 4, p. 19 - 28.

GAMBIER D., VALLADAS H., TISNERAT-LABORDE N., ARNOLD A., Bresson F. 2000 - Datation de vestiges humains présumés du Paléolithique supérieur par la méthode du C14 en SMA. Paléo, 16, p. 1 - 11.

GUTH G. 1973 - Le squelette magdalénien de Saint Germain-la-Rivière. Annales scientifiques de l'Université de Besançon, 18, p. 267 - 274.

HOUËT F., BRUZEK J., MURAIL P. 1999 - Computer program for sex diagnosis in the human pelvic bone based on probabilistic approach. American Journal of Physical Anthropology, suppl. 28, p. 155 (abstract).

HENRY-GAMBIER D., COURTY M. A., CRUBEZY E., KERVAZO K. 2001 - Les enfants de Grimaldi (Grotte des Enfants site des Baoussé - Roussé, Italie). Anthropologie et Palethnologie funéraire. CTHS/RMN.

LUMLEY de M. H., STALENS H., BRUZEK J. 1992 - Nouvelles données sur la sépulture de l'Homme de Menton, Grotte du Cavillon (Baoussé - Roussé, Grimaldi, Italie), Communication à la réunion de la Société préhistorique française, séance de janvier 1992.

MURAIL P., BRUZEK J. et BRAGA J. 1999 - A new approach to sexual diagnosis in past populations. Practical adjustments from Van Vark's procedure. International Journal of Osteoarchaeology, 9, p. 39 $-53$.

PHENICE TW. 1969 - A newly developed visual methods of sexing the Os pubis. American Journal of Physical Anthropology, 30, p. 297 - 301.

SAUTER M. R., PRIVAT F. 1954 - 1955 - Sur un nouveau procédé de détermination sexuelle du bassin osseux. Bulletin de la Société suisse d'Anthropologie et d'Ethnologie, 31, p. 60 - 84.

SCHMITT A. 2001 - Variabilité de la senescence du squelette humain. Réflexion sur les indicateurs de l'âge au décès : à la recherche d'outil performant. Thèse Université Bordeaux I, 347 p. 
SCHULTZ A. H. 1930 - The skeleton of trunk and limbs of higher primates. Human biology, 2, p. 303 $-348$.

SCHULTER-ELLIS F. P., HAYEK L. C., SCHMIDT D. J. 1985 - Determination of sex with discriminant analysis of new pelvic bone measurements: Part II. Journal of Forensic Sciences, 30, p. 178 - 185.

THIEME F. P., SCHULL W. J. 1957 - Sex determination from the skeleton. Human Biology, 29, p. 242 273.

\section{RÉSUMÉS}

Cette note concerne la détermination du sexe du squelette magdalénien de Saint- Germain-laRivière (Gironde) à partir de l'os coxal selon deux méthodes, la première morphologique, la seconde morphométrique dont la fiabilité très élevée a été testée par ailleurs. Cette double analyse indique sans ambiguïté que le squelette de Saint - Germain - la - Rivière appartient à un adulte jeune de sexe féminin.

This paper concerns the sex diagnosis of the magdalenian skeleton from Saint-Germain-laRivière site in Gironde (France). Two methods based on the coxal bone have been used. The first is based on the morphology, the second on the morphometric features. These methods have been tested elsewhere and provide an high accuracy level for sex determination. This analysis shows without ambiguity that the Saint - Germain - la - Rivière individual is a young adult female.

\section{INDEX}

Keywords : Sex diagnosis, os coxal, Homo sapiens sapiens, burial, Magdalenian, Saint-Germainla-Rivière, France

Mots-clés : diagnose du sexe, os coxal, Homo sapiens sapiens, sépulture, Magdalénien, SaintGermain-la-Rivière, France

\section{AUTEURS}

\section{HENRY-GAMBIER}

Université de Bordeaux I - Laboratoire d'Anthropologie des Populations du Passé, UMR 5809 du CNRS - Avenue des Facultés - 33405 Talence

\section{J. BRUZEK}

Université de Bordeaux I - Laboratoire d'Anthropologie des Populations du Passé, UMR 5809 du CNRS - Avenue des Facultés - 33405 Talence

\section{P. MURAIL}

Université de Bordeaux I - Laboratoire d'Anthropologie des Populations du Passé, UMR 5809 du CNRS - Avenue des Facultés - 33405 Talence

\section{F. HOUËT}

Université de Bordeaux I - Laboratoire d'Anthropologie des Populations du Passé, UMR 5809 du CNRS - Avenue des Facultés - 33405 Talence 